\title{
Sistema de Información Web para la Gestión Solicitudes de Jornadas Sociales en Telecomunicaciones por parte de organizaciones públicas y privadas
}

\section{Web Information System for the Management of Social Workshops on Telecommunications by public and private organizations}

Karla Jamileth Cedeño Wheatley

karla jamileth86@hotmail.com

Instituto superior tecnológico Eloy Alfaro

Ecuador

https://orcid.org/0000-0002-1649-2770

Diana Margarita Vera Arroyo

luismariano71119@gmail.com

Instituto superior tecnológico Eloy Alfaro

Ecuador

https://orcid.org/0000-0001-5071-1802

John Wladimir Simisterra Quiñónez

jwsq19821@yahoo.com

Instituto superior tecnológico Eloy Alfaro

Ecuador

https://orcid.org/0000-0002-4628-5406

José Gilberto Argandoña Moreira

joargan@hotmail.com

Instituto superior tecnológico Eloy Alfaro

Ecuador

https://orcid.org/0000-0001-5881-1728

Mariuxi Elizabeth Garcés Wila

mayieli77@hotmail.com

Instituto superior tecnológico Eloy Alfaro

Ecuador

https://orcid.org/0000-0001-7747-4907

Douglerys González

grupodeinvestigaciongik@fundacionkoinonia.com.ve

Fundación Koinonía

Grupo de Investigación Koinonía

Venezuela 


\section{RESUMEN}

El objetivo fundamental de la investigación fue diseñar un sistema de información web para la gestión de solicitudes de jornada y apoyo a venezolana de Telecomunicaciones C.A VTELCA. Como metodología empleada para el análisis y diseño de sistemas de información se utilizó el Manual de creación de aplicaciones de Fábrica VTELCA 2012. Este estudio fue descriptivo, apoyado en una investigación de campo, no experimental, y bajo la modalidad de proyecto factible. La población, objeto de estudio, estuvo constituida por un grupo de 5 personas de diversas gerencias de la fábrica en estudio, a los que se le aplicó un cuestionario en escala con preguntas dicotómicas de 23 ítems, validado mediante juicio de expertos. Los resultados permitieron determinar la necesidad de diseñar de un sistema de información web, con la finalidad de automatizar los procesos de solicitudes de jornadas y donaciones, y ofrecerle a esta organización una herramienta tecnológica mediante la cual se ponga a disposición información de forma rápida, oportuna y segura, y donde a la vez se implementen estrategias que permitirán agilizar todo trámite en relación a las solicitudes en cuanto a jornadas y donaciones sociales por parte de organizaciones públicas y privadas, lográndose así, la simplificación de los procesos administrativos y oportuna respuesta de los mismos.

Descriptores: Sistema de información; Procesos administrativos; Gestión de Solicitudes; Telecomunicación; Tecnología de Información.

\section{ABSTRACT}

The main objective of the research was to design a web information system for the management of working hours and support for Venezuelan Telecommunications C.A VTELCA. As a methodology used for the analysis and design of information systems, the Factory Application Creation Manual VTELCA 2012 was used. This study was descriptive, supported by field research, not experimental, and under the feasible project modality. The population, object of study, was constituted by a group of 5 people of diverse managements of the factory in study, to which a questionnaire was applied in scale with dichotomous questions of 23 items, validated by expert judgment. The results made it possible to determine the need to design a web information system, in order to automate the processes of requests for conferences and donations, and offer this organization a technological tool through which information is made available quickly, in a timely manner and safe, and where at the same time strategies are implemented that will make it possible to expedite all procedures in relation to requests regarding conferences and social donations by public and private organizations, thus achieving the simplification of administrative processes and timely response of same. 
Descriptors: Information system; Administrative processes; Request Management; Telecommunications; Information technology.

Recibido: 10 de mayo del 2019

Aprobado: 15 de junio del 2019

\section{INTRODUCCIÓN}

Las tecnologías de la información y la comunicación (TIC) está produciendo significativos cambios sociales, en especial económicos, ya que la incorporación de estas en una organización puede desencadenar una serie de adaptaciones y procesos, los cuales pueden derivar en mejoras de desempeño que van más allá de los directamente asociados al hecho de contar con un nuevo equipo o herramienta (Bianco y otros, 2002 p.15). Al respecto señala Lattá (2019) que:

Las tecnologías de la información y comunicación, denominadas TIC, han arropado a todas las actividades realizadas por los seres humanos, desde las de orden cotidiana, hasta las de índole laboral, científico, deportivas, culturales, lo que ha conllevado a capacitarse en ese sentido, y hacer de las mismas unas herramientas de trabajo efectivas, en beneficio de las actividades que se realizan (p. 234).

Según Elio Catania, Gerente General de IBM para América Latina (1998): "La creciente importancia de las redes, entre ellas la red suprema (Internet) es el avance (de la informática) más importante de América Latina en los últimos cinco años. El mundo unido por redes cambia la forma de hacer negocios. Incluso cambia la forma en que vivimos, trabajamos, aprendemos y pasamos el tiempo libre. El negocio electrónico es la forma en que describimos como los clientes obtienen una ventaja competitiva mediante tecnologías de redes y la Internet para transformar sus empresas".

Las organizaciones se han visto obligadas a realizar cambios de diseño y métodos de operación a causa de nuevas $\mathrm{Tl}$; antes por ejemplo, la toma de decisiones era muy tardía porque la información y las propuestas tenían que pasar por muchas etapas en la organización antes de que se pudiera decidir o sucediera cualquier cosa. Las TI de hoy 
traspasan varias de esas etapas e incluso permiten la eliminación de algunas de ellas.

Dentro de los cambios realizados en las organizaciones con el apoyo de las tecnologías de información, nacen los Sistemas de Información los cuales han evolucionado según las estrategias empleadas por cada empresa. Sus necesidades y cambios han variado a través del tiempo, según lo han requerido las circunstancias y hechos.

De acuerdo a los criterios establecidos anteriormente nace la necesidad de implicación de nuevas herramientas de tecnología en las organizaciones. Con el propósito de innovación y mejoras encontramos a la Fabrica Venezolana de Telecomunicaciones C.A (VTELCA) la cual nace en el convenio Bilateral entre la República Popular China y La República Bolivariana de Venezuela como Empresa estatal mixta perteneciente al Ministerio de Industrias, Básicas Estratégicas y Socialistas, la cual es la primera ensambladora de equipos celulares y conexos en el país, cuenta actualmente con 5 líneas de producción y se encuentra en construcción la segunda fase.

Como Misión esta empresa busca Impulsar el desarrollo del sistema económico socialista, produciendo, ensamblando y comercializando celulares y otros productos de alta tecnología y óptima calidad que generen felicidad al mayor número de venezolanos y contribuya al fortalecimiento económico y a la soberanía nacional.

Tiene como Visión ser reconocida por todos los venezolanos y a escala internacional como una empresa responsable, productiva y humana, fiel cumplidora de sus compromisos con la población venezolana, con el Estado Venezolano, y con sus trabajadoras y trabajadores. Para el cumplimiento de su visión VTELCA se encuentra estructurada por 12 Gerencia de apoyo y 4 sustanciales, estas últimas mantienen la producción de la Fábrica.

En este sentido, la fábrica desde el 2010 adelanta procesos de transformación que incluyen la creación de la conocida Unidad de Reciclaje para la construcción de objetos utilitarios y juguetes didácticos a partir de la madera que contienen las partes y piezas cuando se internan en el país.

Desde los inicios de la unidad de Reciclaje se construyen juguetes didácticos y bienes utilitarios con las cajas que contienen las partes y piezas de los teléfonos, dándole un 
uso social a la madera para dotar planteles educativos y otros espacios, con el apoyo de personal preparado para este tipo de labor social. (Prensa, VTELCA 2016).

En consecuencia y en cumplimiento de sus vertientes de comercializar equipos de alta calidad y realizar donaciones a Instituciones Educativas, son recibidas constantemente alrededor de 20 a 60 cartas diarias de solicitudes de jornadas y donaciones en la organización por parte de empresas públicas o privadas y Colegios. Información suministrada por el Lcdo. Leswis Romero Jefe de Información de la Gerencia de Asuntos Públicos.

La recepción de estos documentos requiere de un tiempo del personal de la institución, para lo cual se encuentra asignada una sola persona ubicada en el área de recepción de la empresa, la cual atiende a cada una de los solicitantes que llegan a la institución en búsqueda de información, del mismo modo que verificar que las cartas posean correctamente los datos y la información necesaria para la asignación.

Se han manifestado casos en los cuales las personas vienen de diferentes estados del país, y no se les recibe el documento por falta de información o falta del sello si el caso lo amerita, esto implica pérdida de tiempo y de recursos para estas personas que se dirigen la institución. Luego de recibido el documento los solicitantes retornan constantemente a la empresa en seguimiento de sus solicitudes y no reciben respuesta alguna o fecha estimada de la actividad solicitada.

Mientras que, en la organización resulta complejo el manejo diario de todas las cartas recibidas, ya que el personal invierte varias horas hombres en la búsqueda, orden y análisis de cada caso, para luego filtrar la información relevante en algún formato, lo cual puede incurrir en errores a la hora de trascripción de un lado a otro. Por consiguiente aparte de estas actividades se debe prestar la debida atención a todas las personas que se dirigen a la organización en la búsqueda de asesoría para optar a jornadas $u$ donaciones.

Adicionalmente se invierte en espacio para el almacenamiento de todas estas solicitudes, en promedio de veinte cartas diarias, estadísticamente arrojaría alrededor de cuatrocientos mensuales, de la misma forma que conlleva gastos de impresión y 
papelería para él envió de comunicaciones intergerenciales para la atención de estos casos. En relación a estos planteamientos actualmente no se optimizan recursos.

Todo esto planteado anteriormente induce a que no se lleva el control adecuado de este proceso que existe descontento por parte de los clientes, y que para la organización no se optimizan recursos en este proceso. De acuerdo a ello se infiere como solución un sistema de información, donde por medio de una base de datos se obtenga de manera rápida y oportuna lo que se desee consultar. Esta realidad fue evidenciada por el investigador.

La organización no maneja un cronograma de asignaciones de jornadas de ningún tipo, tampoco existe publicación alguna de los requisitos que deben incluir en las cartas emitidas. La organización solo notifica vía telefónica a la institución aprobada la fecha estimada de solicitud, no se considera el tiempo que las personas tomen en dirigirse en busca de respuesta, solo que esperen la llamada, porque tienen una gran cantidad de solicitudes por asignar.

El proceso administrativo para la donación de implementos, involucra directamente al despacho de presidencia de la Empresa VTELCA, específicamente al coordinador adjunto de atención al ciudadano gestión social, y relaciones laborales Rossani Perez, encargada de almacenar la información recibida, y dirigirse a la Gerencia de Gestión Socio-productiva y Relaciones Sociales para solicitar el inventario de implementos escolares, o en su defecto solicitar la creación de los mismos para las instituciones. Realidad evidenciada por el investigador.

Por otra parte, las jornadas de equipos celulares, las asigna la Gerencia de Asuntos Públicos, quienes establecen el estimado de jornadas que se realizaran por periodos establecidos de acuerdo con las solicitudes recibidas, para que no afecte el stock de equipos que posee el agente autorizado de la Institución, y de esta manera confirmar si podrá realizarse la venta de equipos.

Al llevar todo este proceso manualmente se pierde tiempo para organizar, otorgar prioridades, y controlar todas estas solicitudes por tanto se busca mediante un Sistema 
de Información basado en tecnología web automatizar este proceso para lograr, optimizar en tiempos de respuestas las solicitudes, adaptar la institución a cambios tecnológicos, mejorar la gestión. Así como también la efectividad en la toma de decisiones, tomando en cuenta criterios de eficacia, calidad y equidad que son factores estratégicos para los servicios de atención para ofrecer a la comunidad en general los mejores servicios.

Del mismo modo que permitirá a la directiva de la institución evaluar que se cumplan con el rol de la empresa de llevar a las comunidades de manera directa las jornadas, considerando también que se podrá identificar a donde fueron enviados los recursos didácticos desarrollados por el taller de reciclaje de la empresa. Esto a través de reportes que se generan mediante la aplicación web, los cuales sirven de insumo para estadísticas de logros que apoyen el avance de la organización.

Es importante mencionar que el sistema de Información que se desea incluir es de tipo estratégico; ya que busca la interacción con el entorno, cuyo propósito se encuentra en buscar ventajas competitivas y establecer relaciones con el cliente (Cohen y Asín, 2000). En este caso la comunidad en general que realizan solicitudes a la organización, facilitando el trabajo de ambas partes en disponibilidad, accesibilidad, control y responsabilidad.

Resumiendo lo antes expuesto se busca el cambio tecnológico y oportunidad de mejora en los procesos de la organización, inclusive los altos directivos de la organización podrán visualizar en tiempo real las actividades que realiza la empresa, las solicitudes que poseen activas, todo con respecto a la atención tanto a comunidades, como a planteles educativos u otras instituciones que soliciten de estos servicios.

Para el cumplimento de esta investigación surgieron las siguientes interrogantes relacionadas con el diseño de un sistema de Información para este proceso de solicitudes dentro de la organización:

- ¿Cuál es la situación actual para la asignación de jornadas y para el proceso de 
donación de implementos escolares?

- ¿Cuáles son los mecanismos de validación de las instituciones solicitantes para verificar su validez?

- ¿Cuáles requerimientos técnicos, metodológicos y tecnológicos ayudaran al diseño del sistema?

La búsqueda de respuestas a estas interrogantes forma el centro de la investigación, ya que serán los elementos cruciales que ayudarán, para el diseño del Sistema de Información, como una alternativa viable que garantice la fiabilidad y accesibilidad, así como también la toma de decisiones en pro de convertir a VTELCA en una empresa innovadora que apoya sus procesos administrativos con la tecnología.

\section{Objetivos de la investigación}

Diseñar un sistema de información web para la gestión de solicitudes de jornadas de equipos celulares y apoyo de donación de implementos escolares, para la Empresa Venezolana de Telecomunicaciones C.A VTELCA.

\section{METODOLOGÍA}

De acuerdo al criterio sustentado por Hernández y otros (2006, p.205), la investigación se inserta dentro del diseño no experimental, ya que ésta se realizó "sin manipular deliberadamente las variables", se buscó la observación del fenómeno en estudio tal como se da en su ambiente natural, recolectando la información en un momento único, buscando lograr la descripción de la variable y el análisis de su incidencia en un determinado tiempo. Asimismo, la investigación se enmarcó en un estudio de campo, porque los datos de interés se recogieron en forma directa de la realidad donde se desarrolló la problemática objeto de estudio.

La investigación es de naturaleza descriptiva, porque se expone el evento estudiado 
enumerando detalladamente sus características, se registran, analizan e interpretan la totalidad de los datos suministrados por la muestra. Es un tipo de investigación asociada al diagnóstico, siendo que, en la investigación de tipo descriptivo:

...el propósito es exponer el evento estudiado, haciendo una enumeración detallada de sus características, de modo tal que en los resultados se pueden obtener dos niveles de análisis, dependiendo del fenómeno y del propósito del investigador: un nivel más elemental, en el cual se logra una clasificación de la información de función de características comunes, y un nivel más sofisticado en el cual se ponen en relación los elementos observados a fin de obtener una descripción más detallada. (Hurtado 2006, p.103)

Asimismo, se enmarca en la modalidad de proyecto factible, dado que su propósito se encuentra en Diseñar un Sistema de Información Web para la gestión de solicitudes de Jornada y apoyo a Venezolana de Telecomunicaciones VTELCA, por tanto se define un proyecto factible en vista de que se considera una propuesta de acción para resolver un problema, orientado a satisfacer una necesidad existente (UPEL, 2004, p.16).

\section{RESULTADOS}

En atención al objetivo planteado, a la problemática expuesta y una vez aplicado el cuestionario a los responsables y actores principales del proceso de asignación de jornadas y donaciones de implementos escolares, además de contribuir con la descripción de los procesos administrativos de las solicitudes que se tramitan en la fábrica, permitió determinar la necesidad de implementar una estrategia, como lo son los sistemas de información web, con lo cual se podría mejorar la eficiencia en sus procesos y facilitar el trabajo a la organización con apoyo de la tecnológica a la vez que brindar un servicio de calidad a los solicitantes.

\section{PROPUESTA}

\section{Introducción}

La globalización de la economía da origen a más riesgos pero también a más oportunidades para todos y obliga a las organizaciones a efectuar cambios 
permanentes para poder prosperar, mantenerse competitivos o simplemente sobrevivir. Es por ello que existen avances tecnológicos que originan una cantidad importante de nuevas herramientas que obligan a las organizaciones a su adquisición para incluirlas en el desarrollo de sus procesos con la finalidad de mantenerse competitivas en un mercado permanentemente cambiante y cada vez más saturado.

Las organizaciones no pueden ser conservadoras cuando se trata de la optimización de sus procesos por medio de la inclusión de nuevas herramientas tecnológicas. Estas tecnologías deben encontrarse fundamentalmente en las áreas estratégicas; sin embargo, es importante que todas las unidades de la organización se encuentren orientadas en la misma línea tecnológica. El éxito de una empresa depende de la calidad de su información y de la velocidad con la que ésta puede ser comunicada.

Para lograr optimizar el flujo de información es necesario automatizar los procesos administrativos a través de los sistemas de información los cuales entre diversas ventajas ponen a disposición de quien lo demande y en el momento en que se requiera toda la información, reduciendo el tiempo y facilitando la realización de cualquier trámite.

Para el cumplimiento de esta investigación se propone la automatización del proceso de asignación de jornadas de equipos y donación de implementos escolares de Venezolana de Telecomunicaciones C.A VTELCA, con el fin de ofrecerle una herramienta tecnológica mediante la cual tengan a su disposición la información de manera rápida y oportuna, y a su vez se permita agilizar este proceso de solicitudes de forma más fácil y cómoda tanto para los entes que deseen adquirir algún beneficio como para la misma organización.

\section{Justificación de la Propuesta}

El uso masivo de las TIC en el funcionamiento diario de las organizaciones se ha generalizado. La capacidad de definición y gestión de una estrategia TIC acorde con los objetivos y la estructura organizativa de una institución se ha transformado en una obligación inexcusable para su personal directivo. Dado esto el mundo empresarial se 
hace cada vez más competitivo dentro de su ramo y constantemente adoptan cambios tanto administrativos como tecnológicos que cubran sus necesidades a fin de garantizar el logro de sus metas.

Sin duda alguna, estas organizaciones deben apoyarse en los sistemas de información, los cuales generan datos precisos para ayudar a la toma de decisiones y la resolución de problemas de forma oportuna e inteligente. Los cuales actualmente permiten llevar acabo procesos manuales de una forma automatizada, reduciendo altos costos y retrasos en los procesos.

Debido a ello es de suma importancia la inclusión de los mismos dentro de los procesos administrativos de las organizaciones, como es el caso de este proceso que actualmente se encuentra débil y con la necesidad de inclusión de nuevas tecnologías ya que entre diversos motivos existen retrasos en las comunicaciones, en las respuestas a los solicitantes, perdida de horas hombres por ordenar la cantidad de papeles, gastos innecesarios en espacios de almacenamiento.

Es por ello, que el sistema de información propuesto permitirá que la toma de decisiones se efectúe oportunamente en tiempo y forma, facilitando el direccionamiento de las solicitudes, considerando un orden de prioridad y la detección oportuna de desviaciones para aplicar los correctivos que sean necesarios, lográndose así, optimizar el proceso completo.

\section{Ubicación Administrativa}

Para el desarrollo de esta investigación las áreas objeto de estudio son aquellas que se encuentra vinculadas directamente con el proceso a automatizar como lo es Despacho de Presidencia, Gerencia de Relaciones Socialistas y Gerencia de Asuntos Públicos de Venezolana de Telecomunicaciones C.A VTELCA, ubicada en la Zona Franca Industrial de Paraguana, Punto Fijo - Estado Falcón.

\section{Objetivo General}

Automatizar el proceso de asignación de Jornadas de Equipos y Donación de 


\section{Objetivos Específicos}

Optimizar los procesos desglosados de la asignación de jornadas de equipos y asignación de jornadas de implementos escolares (solicitudes, aprobación, fechas programadas).

Gestionar datos e información solicitada por los clientes o beneficiado de este proceso.

Minimizar la pérdida de tiempo y de recursos materiales que permitan la agilización y el ahorro durante la gestión administrativa.

Establecer mecanismos de seguimiento y control de las solicitudes por parte de los entes solicitantes.

\section{Factibilidad de la Propuesta}

Se conoce como factibilidad al grado en el que lograr algo es posible o las posibilidades que tiene de lograrse. Con el propósito de determinar la viabilidad de esta propuesta fue necesario tomar en consideración ciertos factores que permitieron establecer los beneficios y costos en caso que el sistema se implantará; estos factores son: factibilidad legal, organizacional, económica, técnica y operacional.

\section{Factibilidad Organizacional}

Esta factibilidad está a cargo de los encargados del proceso, personal de Fábrica VTELCA de las áreas de Relaciones Socialistas, Asuntos públicos, y despacho de Presidencia de la misma. En su mayoría Licenciados en administración.

\section{Factibilidad económica}

Los recursos que se consideraron en esta factibilidad son el costo en hora/hombre 
invertidas en el diseño de la aplicación, así como el costo de la plataforma hardware/software necesarios, los cuales fueron cubiertos por la autora de esta investigación.

\section{Factibilidad técnica}

Consiste en establecer los recursos técnicos actuales que puedan ser mejorados o adquiridos para la implementación del nuevo sistema. Es decir, el análisis de la factibilidad técnica permite evaluar si el equipo y software están disponibles y si cumple con las especificaciones técnicas requeridas por cada alternativa del diseño que se está considerando.

Este estudio dio como resultado que en la Gerencia de tecnología de Información y Comunicación de fábrica VTELCA se dispone de los recursos de hardware y software para alojar la aplicación. El hospedaje Web requiere un servidor Web, con soporte para PHP y el Manejador de Bases de Datos MySQL, el cual puede almacenar bases de datos, permitiendo que el sistema propuesto garantice la confiabilidad y seguridad de la información.

\section{Factibilidad Operacional}

Considera si la organización cuenta con el personal con la experiencia técnica, manejo y destreza requerida para manejar el sistema propuesto. En este casos se puede decir que el personal posee experiencias en el manejo de aplicaciones por tanto es fácil para ello adaptarse a herramientas tecnológicas.

\section{Sistema de Información Web para la Gestión de Solicitudes de jornada y donaciones a Venezolana de Telecomunicaciones C.A. VTELCA}

Para la creación del Sistema se utilizó la metodología propia de desarrollo de aplicaciones de fábrica VTELCA según el Manual de creación de aplicaciones Fábrica VTELCA de 2012. Dicha metodología propone 4 fases para el desarrollo de sistemas de información estas son: identificación de los requerimientos, diseño conceptual de los 
requerimientos (diseño de la propuesta, de la navegación y de la interfaz) generación de código, pruebas e implementación.

Cada una de estas fases identifica cada proceso en el desarrollo del sistema. Es importante señalar que para esta propuesta, se llevó a cabo el desarrollo hasta la tercera (3) fase que hace referencia al diseño del sistema recomendado, definido como objetivo general del estudio.

\section{1.- Identificación de los Requerimientos}

Esta fase es la etapa inicial del proyecto, y es la principal que determinara el éxito del desarrollo del mismo, requiere que se observe de forma objetiva lo que ocurre en una organización, luego en conjunto con otros miembros de la organización hacer notar los problemas. Durante esta fase se debe conocer las necesidades de los usuarios, los actores involucrados en la misma, el tipo de desarrollo y si existe participación de las áreas externas.

Para determinar los requerimientos de información dentro de una organización pueden utilizarse diversos instrumentos, los cuales incluyen: muestreo, el estudio de los datos y formas usadas para la organización, la entrevista, los cuestionarios; la observación de la conducta de quien tomó la decisión, así como de su ambiente. Se hace todo lo posible por identificar qué información requiere el usuario para desempeñar sus tareas.

En este caso en particular, el problema fue identificado inicialmente por inquietudes mencionadas por algunas personas que se acercaban a la institución en busca de jornada de equipos, y por directivos de instituciones escolares que deseaban los

implementos escolares para mejoras en las aulas de clases de los niños. Posterior a ello se comenzó el seguimiento de como se manejaba este proceso internamente y porque no había respuesta en un tiempo prudencial, donde se observó que se manejaban grandes volúmenes de solicitudes que dificultaban el análisis de cada una de manera rápida y por consiguiente asignar el orden de prioridades para su posterior aprobación, lo cual dio inicio a esta investigación.

Es importante resaltar, que para este estudio se empleó una encuesta escrita tipo 
cuestionario, a fin de corroborar y constatar dicha problemática, la cual arrojó como resultado que las áreas en estudio, no disponen de una adecuada y sistematizada organización en cuanto a estas solicitudes, lo que dificulta realizar el seguimiento y control respectivos para los altos directivos, e impide dar respuesta rápida u oportuna a los solicitantes.

Ante tal situación, resulta realmente oportuno disponer de una herramienta informática que le permita a los solicitantes realizar sus solicitudes desde la comodidad de su hogar, así como conocer en línea los requisitos que deben cumplir para optar a estos beneficios, y desde la organización mantener un control sistemático de las solicitudes que se van generando y manejar la misma información todas la gerencias involucradas. Así mismo permitirá a los altos directivos generar un reporte en cualquier momento, de las solicitudes procesadas y atendidas en un periodo de tiempo determinado, lo cual apunta mas adelante a evaluar el proceso, al personal con el fin de ir mejorando sustancialmente aplicando mejores estrategias con el apoyo de estas herramientas tecnológicas.

De acuerdo al cuestionario se permitió recabar y analizar todos los datos necesarios para lograr determinar con exactitud qué tipo de información requería el sistema propuesto. Luego de ello se llenaron dos matrices de requerimientos para el sistema de información propuesto, una donde se especifica los módulos donde trabajaran los usuarios externos, y otra para los módulos de personal administrativo de fábrica VTELCA, como se observa a continuación: 


\section{Matriz de Requerimientos 1.- Usuarios Externos} Gojiamo Bolivariano
de venezuvila

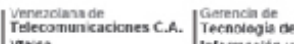

Tecnolegis a

\section{MATRIZ DE REQUERIMIENTOS}

\begin{tabular}{|c|c|c|c|c|c|}
\hline & & Id de Matriz: & MR-SGS200120 & & \\
\hline & & Autor(a): & Ing. Douglerys G & alez & \\
\hline & & Desarrollador: & & & \\
\hline & & Fecha: & $20 / 01 / 2017$ & & \\
\hline & & ID Proyecto: & & & \\
\hline & & Usuario: & Externos & Firma: & \\
\hline & & Usuario: & Organizaciones & Firma: & \\
\hline & & Gerente/Supervisor: & Ysabel Zarraga & Firma: & \\
\hline ID & & EQUERIMIENTO & DS DEL USUA & & \\
\hline - REQL & RIMIENTOS DE CALIDAD & E SOFTWARE: & & & \\
\hline RQ-01 & Implementar un Servicio de A & tenticación de Usuario & & & \\
\hline RQ-02 & Realizar Documentación Técr & ca de la Aplicación. & & & \\
\hline RQ-03 & Con figurar Usuario para prue & as y Validación de Reg & querimientos. & & \\
\hline RQ-04 & Realizar el Manual de Usuari & de la Aplicación. & & & \\
\hline RQ-05 & Implementar los Estándares C & áficos de la Gerencia de & le asuntos Públicos & & \\
\hline - REQL & RIMIENTOS TÉCNICOS: & & & & \\
\hline RQ-06 & Utilizar el Lenguaje de Progr & mación PHP para la coc & dificación. & & \\
\hline RQ-07 & Implementar como manejador & e Base de Datos Mysql & & & \\
\hline - REQL & RIMIENTOS DE USUARIO & INAL: & & & \\
\hline RQ-08 & $\begin{array}{l}\text { Incorporar una opción de hip } \\
\text { Solicitudes. }\end{array}$ & vínculo dentro de la pa & agina oficial de $f_{c}$ & Ca VTEL & EA para la Gestión de \\
\hline RQ-09 & Realizar una ventana de notific & ción de los requisitos qu & ue deben cumplir & 1 registrar & la solicitud. \\
\hline RQ-10 & Realizar un modulo para el reg & tro de usuario para el re & establecimiento de & atraseñas. & \\
\hline RQ-11 & Realizar un modulo para el reg & tro de solicitud de equip & pos o donaciones & mplement & os escolares. \\
\hline RQ-12 & Realizar un modulo para la cor & ulta de solicitudes y estic & atus de la mismas & & \\
\hline RQ-13 & Realizar un modulo para impri & ír planilla de solicitud. & & & \\
\hline
\end{tabular}




\section{ESPECIFICACIÓN DE REQUERIMIENTOS}

ID-Requerimiento: RQ-01.

\section{Descripción Detallada: Servicio Autenticación de Usua rios}

El Sistema debe implementar un Servicio de Autenticación, Autorización y Auditoría para el manejo de los usuarios. Premisas:

El Sistema debe permitir Gestionar Usuarios.

El Sistema debe manejar de forma correcta el Proceso de Autorización.

El Sistema debe manejar un proceso de Auditoría mediante la implementación de este Servicio.

ID-Requerimiento: RQ-02

Descripción Detallada: Documentación Técnica.

El sistema debe poseer la documentación técnica de la codificación, con el fin de comprender cada uno de los procedimientos, métodos, funciones, clases, entre otros, realizados por el prog ramador.

\section{Premisas:}

Sin premisas.

ID-Requerimiento: RQ-03

Descripción Detallada: Usuario para pruebas y Validación de Requerimientos.

Dent ro del proceso de desarrollo se requiere realizar el seguimiento y validación de requerimientos descritos por el usuario final, para ello se necesita que la aplicación cuente con la configuración de un usuario para realizar las pruebas pertinentes.

\section{Premisas:}

El usuario debe tener todos los privilegios.

\section{ID-Requerimiento: $\mathrm{RQ}-04$}

\section{Descripción Detallada: Realizar el Manual de Usuario de la Aplicación}

El Sistema debe poseer el manual usuario, con el fin de que el mismo pueda comprender todas las funcionalidades de la aplicación y darle el uso correcto al sistema.

Premisas:

Debe ser realizado con el formato para tal fin proporcionado por el Departamento de Desarrollo y Aplicaciones. 
ID-Requerimiento: RQ-05

Descripción Detallada: Implantar los Estándares Gráficos de la Gerencia de asuntos Públicos de Fábrica VTELCA

El Sistema debe implementar los estándares gráficos proporcionado por loa Gerencia de asuntos públicos de la Fábrica Vtelca para realizar la interfaz gráfica de la aplicación.

Premisas:

Sin premisas

ID-Requerimiento: RQ-06

Descripción Detallada: Utilizar el Lenguaje de Programación PHP para la codificación

Es necesario utilizar un lenguaje de código abierto para cumplir con el decreto 3390 sobre en el uso de Software Libre en la Administración Pública. Se recomienda emplear PHP para la codificación del sistema.

Premisas:

Sin Premisas

ID-Requerimiento: $\underline{R Q-07}$

Descripción Detallada: Implemen tar como manejador de Base de Datos Mysql.

Es necesario utilizar esta herramienta ya que VTELCA, posee todas las aplicaciones con es te manejador de base de datos. Premisas:

Sin Premisas

ID-Requerimiento: $\mathrm{RQ}-08$

Descripción Detallada: Incorporar una opción de hipervínculo dentro de la pagina oficial de fábrica VTELCA para la Gestión de Solicitudes.

Se solicita que dentro de la pagina de fábrica VTELCA, www.vtelca.gob.ve se asigne una opción denominada gestión de solicitudes que redireccione al sistema de solicitudes.

Premisas:

Sin premisas.

ID-Requerimiento: RQ-09

Descripción Detallada: Realizar una ventana de notificación de los requis itos que deben cumplir para registrar la solicitud.

La misma debe ser una ventana emergente que muestre un mensaje de advertencia.

Premisas:

Sin premisas.

ID-Requerimiento: $\underline{\mathrm{RQ}-10}$

Descripción Detallada: Realizar un modulo para el registro de usuario y para el res tablecimiento de contraseñas. 
Descripdón Detallada: Realizar un modulo para el regist vo de soliciud de equipos o donaciones de implementos escola ves

En este modulo deben seleccionar primeramente el tipo de solicitud de acuerdo a ello se mostrara para donaciones de implementos escolares o para Jomada de equipos.

Premisas: Debe mostrar primeramente el inventario de equipos para la solicitud de jornadas.

ID-Requerimiento: RQ-12

Descripdón Detallada: Realizar un modulo para la consılta de solicitudes y estatus de la mismas

En este modulo se debe mostrar las solicitudes que tengan activa los usuarios y el estatus en que se encuentre la misma recibido, aprobado y en proceso de asignación de fecha.

Premisas:

ID-Requerimiento: RQ-13

Descripdón Detallada: Realizar un modulo para imprimir planilla de solicitud.

En este modulo se debe generar el pdf de la solicitud generada.

Premisas: 


\section{Matriz de Requerimientos 2.- Usuarios Personal Administrativo Fábrica VTELCA}

\section{MATRIZ DE REQUERIMIENTOS}

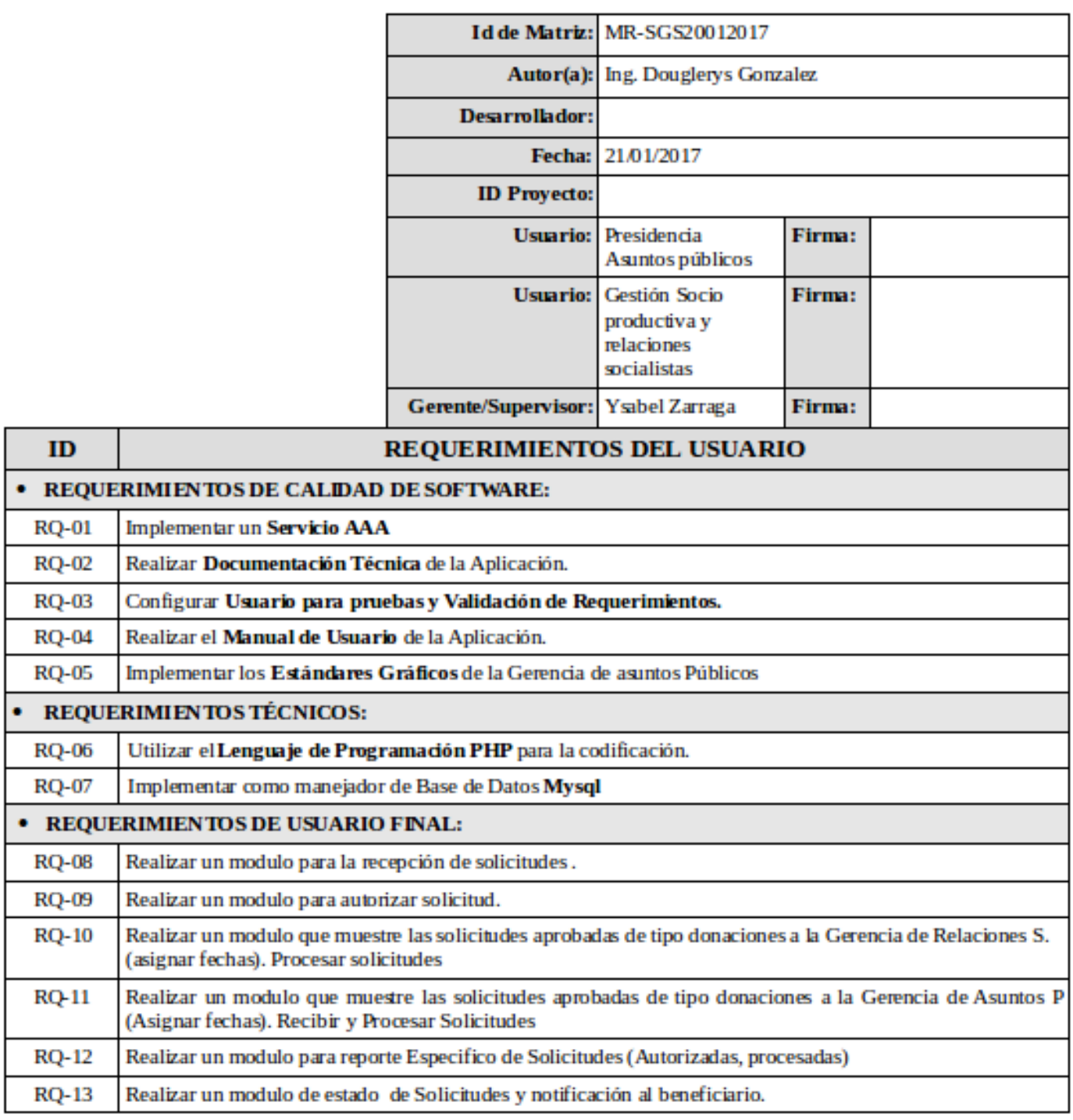




\section{ESPECIFICACIÓN DE REQUERIMIENTOS}

ID-Requerimiento: RQ-01.

\section{Descripción Detallada: Servicio AAA}

El Sistema debe implementar un Servicio de Autenticación, Autorización y Auditoria para el manejo de los usuarios.

\section{Premisas:}

El Sistema debe permitir Gestionar Usuarios según permisologia.

El Sistema debe manejar de forma correcta el Proceso de Autorización.

El Sistema debe manejar proceso de Auditoria mediante la implementación de este Servicio.

\section{ID-Requerimiento: RQ-02}

\begin{tabular}{l} 
Descripción Detallada: Documentación Técnica. \\
$\begin{array}{l}\text { El sistema debe poseer la documentación técnica de la codificación, con el fin de comprender cada uno de los } \\
\text { procedimientos, métodos, funciones, dases, entre otros, realizados por el programador. }\end{array}$ \\
\hline Premisas: \\
\hline Sin premisas.
\end{tabular}

ID-Requerimiento: RQ-03

\section{Descripción Detallada: Usuario para pruebas y Validación de Requerímientos.}

Dentro del proceso de desarrolb se requiere realizar el seguimiento y validación de requerimientos descritos por el usuario final, para ello se necesita que la aplicación cuente con la configuración de un usuario para realizar las pruebas pertinentes.

\section{Premisas:}

El usuario debe tener todos los privilegios.

ID-Requerimiento: RQ-04

\section{Descripción Detallada: Realianr el Manual de Usunrio de la Aplicación}

El Sistema debe poseer el manual usuario, con el fin de que el mismo pueda comprender todas las funcionalidades de la aplicación y darle el uso correcto al sistema.

\section{Premisas:}

Debe ser realizado con el formato para tal fin proporcionado por el Departamento de Desarrollo y Aplicaciones. 
ID-Requerimiento: RQ-05

ID-Requerimiento: RQ-06

Descripción Detallada: Utiliar el Lenguaje de Programación PHP para k codificación

Es necesario utilizar un lenguaje de código abierto para cumplir con el decreto 3390 sobre en el uso de Software Libre en la Administración Pública. Se recomienda emplear PHP para la codificación del sistema.

\section{Premisas:}

Sin Premisas

ID-Requerimiento: $\mathrm{RQ}-07$

Descripción Detallada: Implementar como manejador de Base de Dabs Mysql

Es necesario utilizar esta herramienta ya que VTELCA, posee todas las aplicaciones con este manejador de base de datos. Premisas:

Sin Premisas

ID-Requerimiento: RQ-08

Descripción Detallada: Realizar un modulo para la recepción de solicitudes.

Se solicita que se realice un modub que permite visualizar todas las solicitudes y cambiar su estado a recibido.

Premisas:

Depende de las solicitudes registrada por los usuarios.

ID-Requerimiento: $\mathrm{RQ-09}$

Descripción Detallada: Realizar un modulo para autorizar solicitud.

Se solicita se realice un modulo que permita autorizar la solicitud antes de proceder a la ejecucción de la misma.

Premisas:

Sin premisas.

ID-Requerimiento: $\mathrm{RQ}-10$

Descripción Detallada: Realizar un modulo que mueș re las solicitudes aprobadas de típo donaciones a la Gerencia de Reladiones S.

El modulo debe permitir mostrar las solicitudes despues de autorizadas donde el usuario pueda colocar procesadas y fecha 
ID-Requerimiento: $\mathrm{RQ}-11$

\section{Descripción Detallada: Realizar un modub que muestre las solicitudes aprobadas de tipo donaciones a la Ge rencia} de Asuntos Püblicos

El modulo debe permitir mostrar las solicitudes después de autorizadas donde el usuario pueda colocar procesadas y fecha de realización de la jornada.

Premisas:

ID-Requerimiento: RQ-12

\section{2.- Diseño Conceptual de los Requerimientos}

Esta fase se encuentra dividida en 3 sub- fases ya que abarca un largo contenido en lo que se refiere a ciclo de vida de los sistemas de información, para dar continuidad a la investigación a continuación se presentan la forma en cómo se abordaron las mismas.

\section{a.- Diseño de la Propuesta}

Para el diseño de la propuesta se realizaron diagramas que permiten visualizar la interacción propuesta según los nuevos requerimientos de los usuarios, el mismo se 
representó mediante diagramas de carretes según los estándares indicados por el Departamento de Aseguramiento de la Calidad quien es el responsable de estandarizar los documentos de procesos, de la misma forma en que se realizaron dos matrices de requerimientos separando los externos y el personal administrativo; asímismo se realiza con los diagramas. 


\section{Diagrama 1.- Proceso de usuarios Externos}
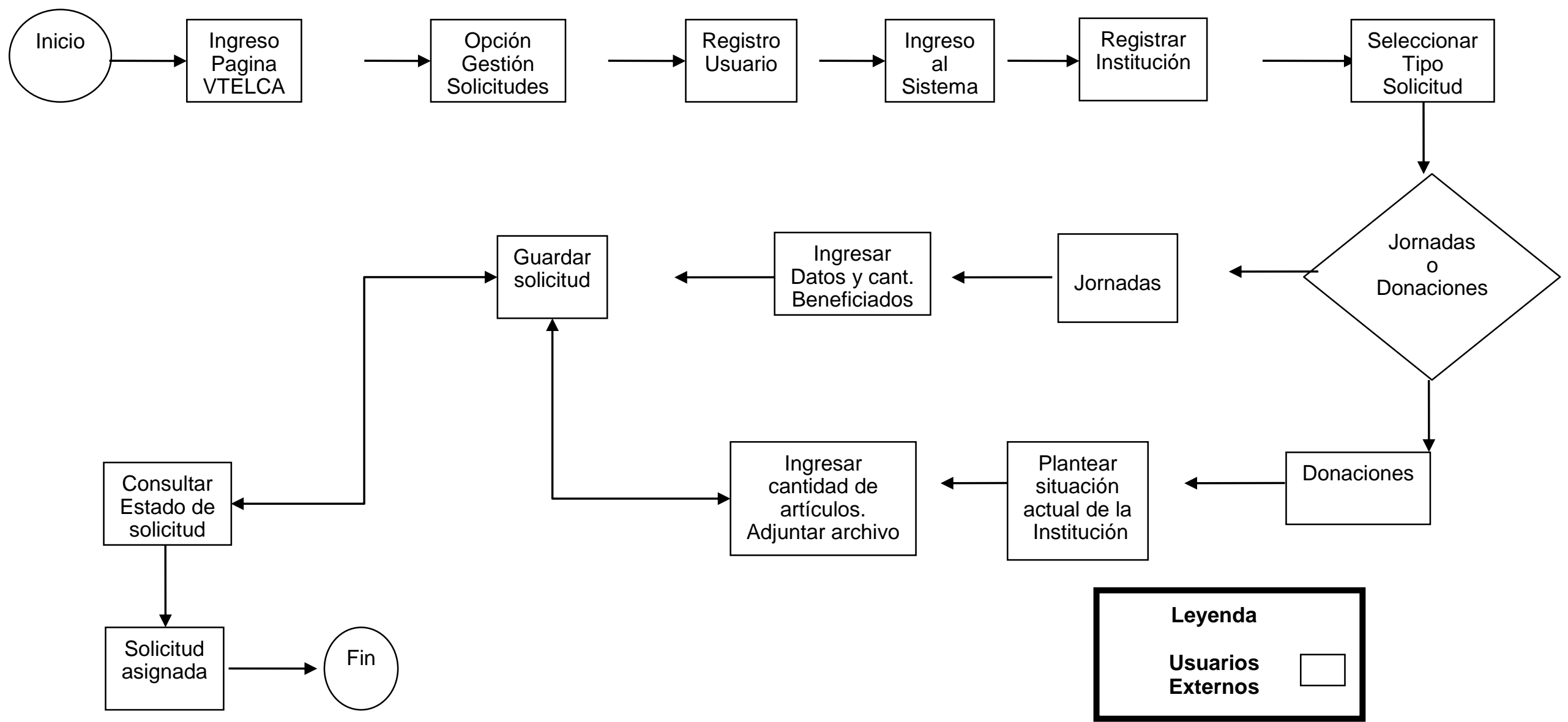

Ingresar cantidad de

artículos.

Adjuntar archivo
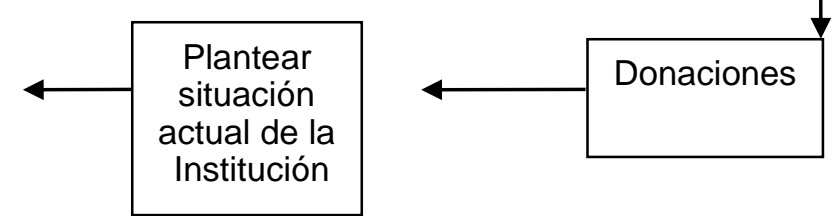

Leyenda

Usuarios

Externos 
Walter Bermudez; Luis Urueta

\section{Diagrama 2.- Proceso de Personal Administrativo}
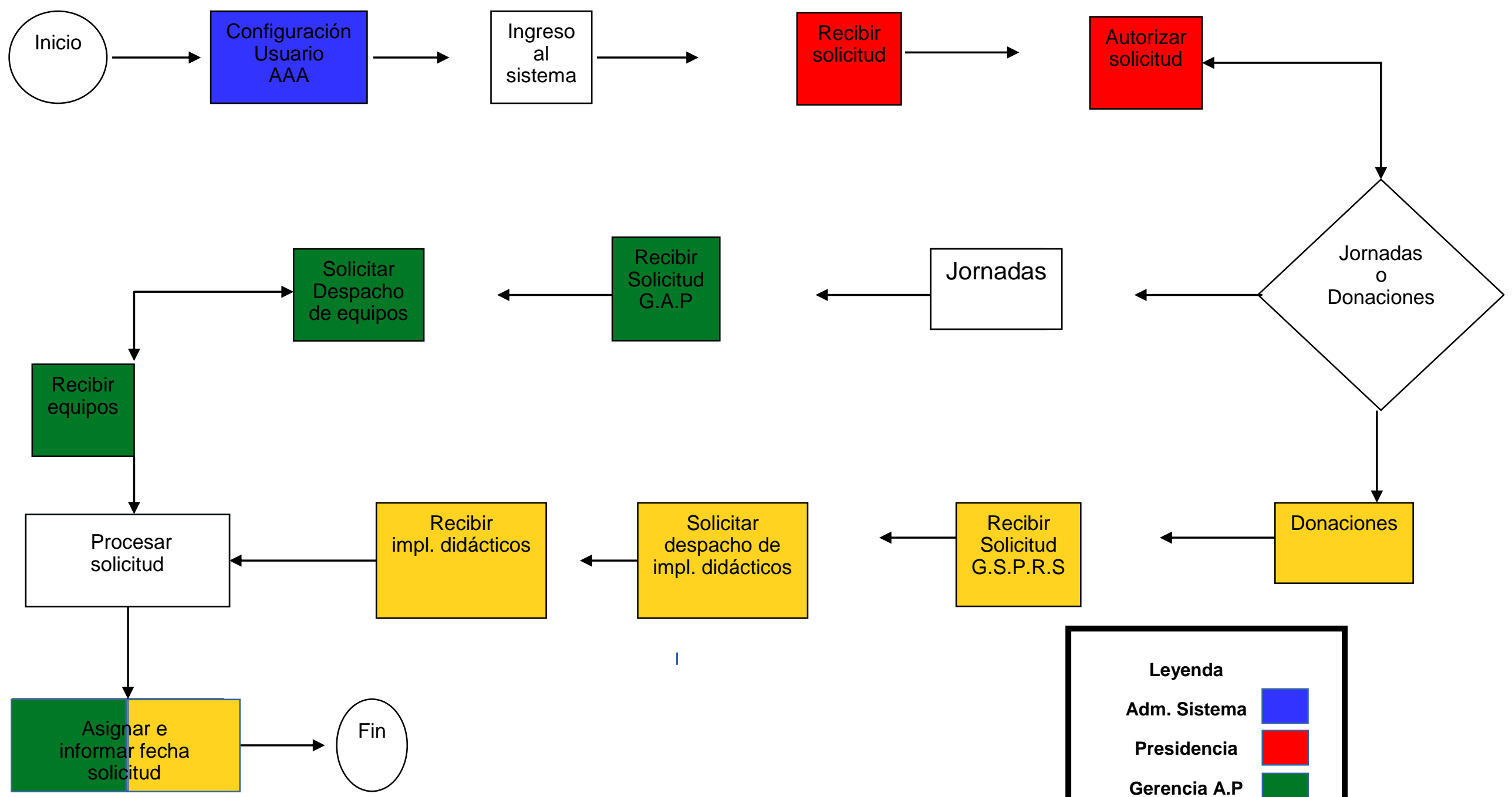

Leyenda

Adm. Sistema

Presidencia

Gerencia A.P 


\section{b.- Diseño de Navegación}

El diseño de navegación debe comenzar con la jerarquía de usuarios y el tipo de uso relacionado con cada categoría definida (actor), se debe tener presente que cada actor puede usar la aplicación de manera un poco diferente, y por tanto, puede tener diferentes requisitos de navegación. En este sentido, culminado este diseño deberá discutirse con el usuario para su aprobación.

\section{Diagrama 3.- Diseño de navegación Usuarios Externos}

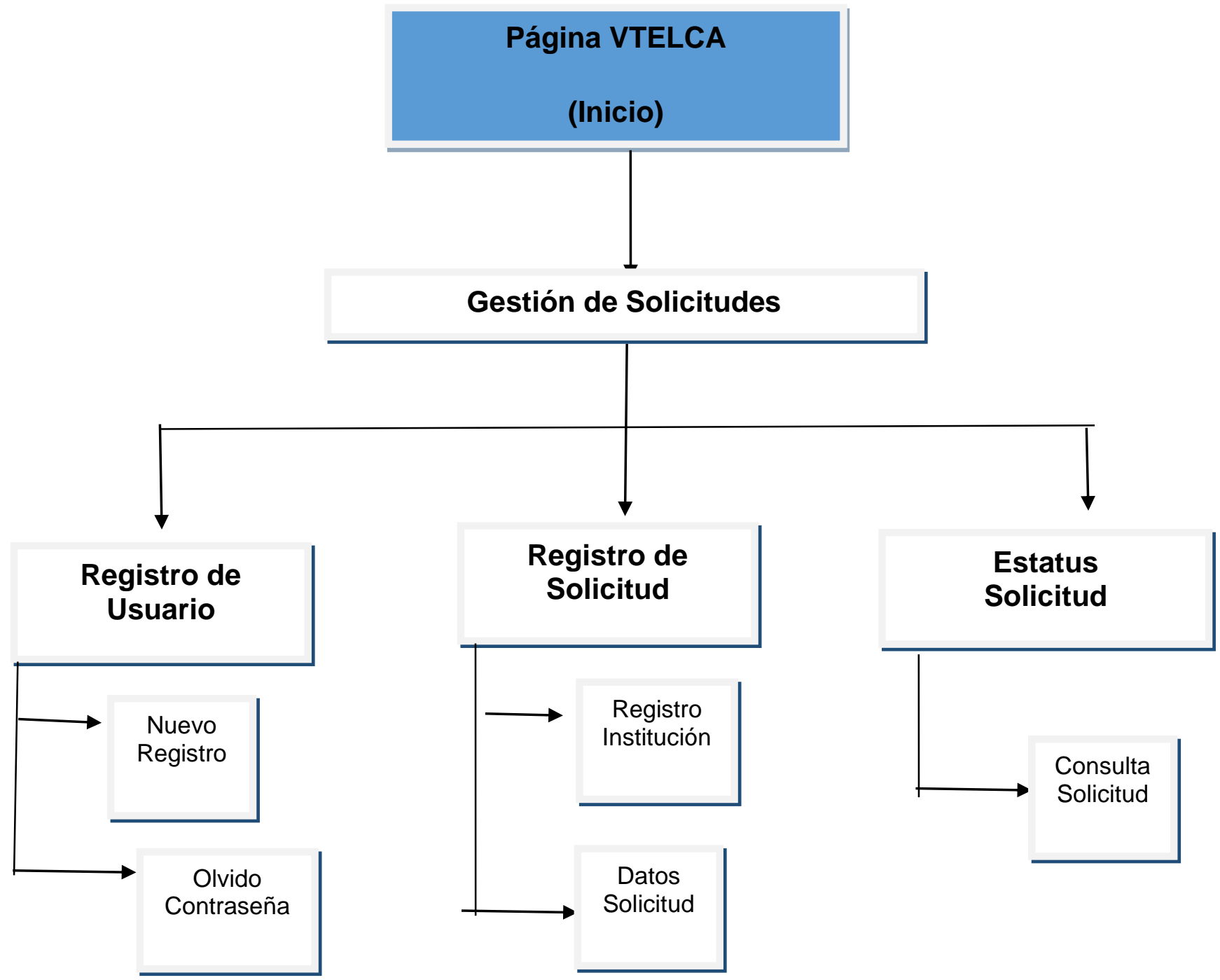




\section{Diagrama 4.- Diseño de Navegación}

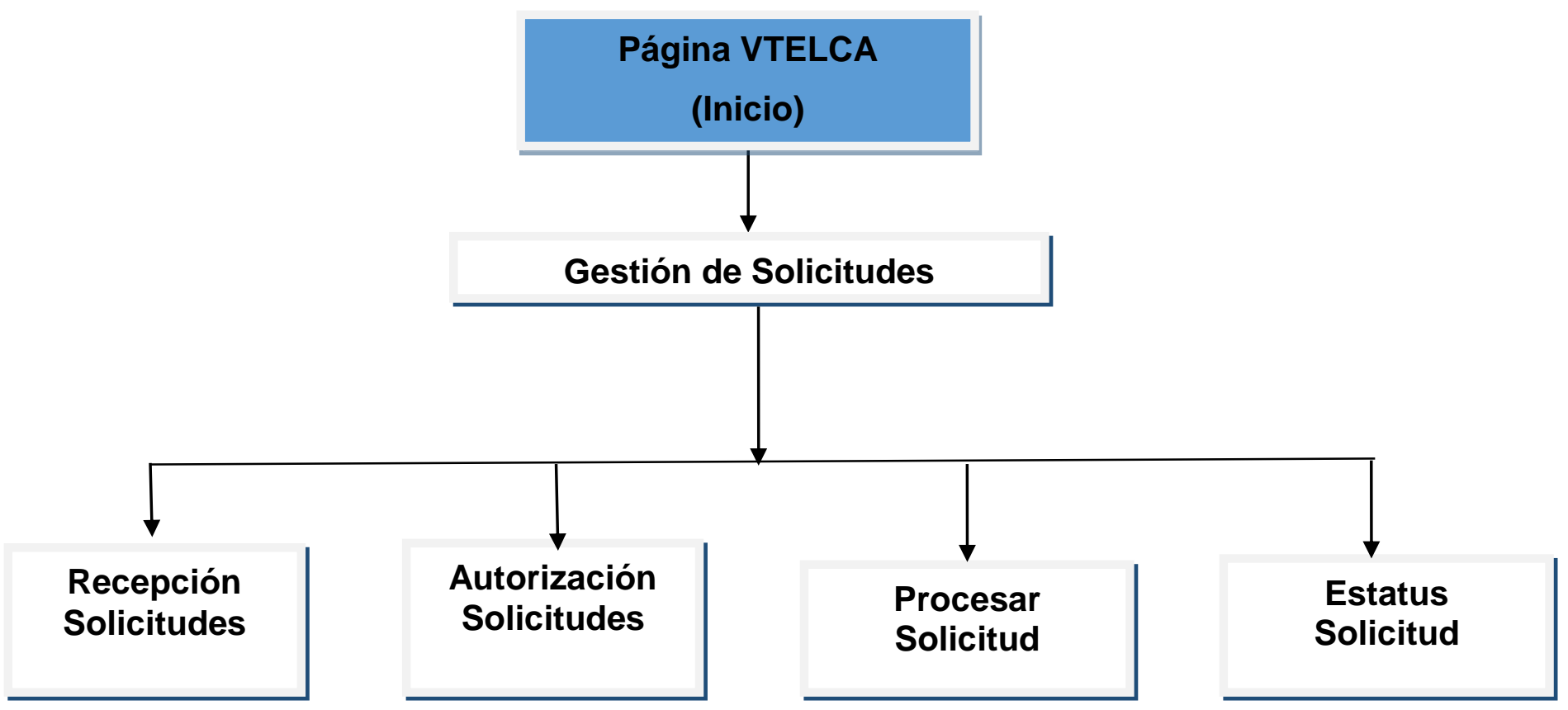

\section{C.- Diseño de Interfaz}

Para el diseño de las pantallas que conformaron el requerimiento solicitado, se tomó en cuenta los estándares visuales pautados por la Gerencia de Asuntos Públicos de VTELCA, en relación a la ubicación y constitución de la cabecera y pie de pagina de cada formulario y/o reportes.

Durante el proceso de diseño de la interfaz gráfica se usó los recursos visuales establecidos y disponible en la siguiente dirección: www.rec.vtelca.gob.ve, para la representación de los botones e imágenes a insertar para la simulación de las acciones, a considerar dentro del desarrollo del diseño, el cual deberá basarse en todo momento en la plantilla de desarrollo propia del Departamento de aplicaciones, para garantizar el mantenimiento efectivo de las aplicaciones por cualquier custodio en el tiempo.

Se establecieron los módulos que componen el sistema de información, con el fin de organizarlos en una estructura accesible al usuario. En este sentido se pueden mencionar los siguientes módulos del sistema: 


\section{Usuario: Externos (Personal Solicitante)}

- Pagina VTELCA (hipervínculo)

- Registro de Usuario (1.- usuarios registrados, registrarse)

- Registro de Institución

- Registro de Solicitud

- Consulta de Solicitud

- Cerrar Sesión

\section{Usuario: Personal Administrativo Fábrica Vtelca}

- Recepción de Solicitud

- Autorización de Solicitud

- Procesar Solicitudes

- Consulta Solicitud

Es importante mencionar que para el control de acceso se establece por fábrica VTELCA, el uso del servicio AAA, Autenticación, Acceso y Auditoria de sistemas.

\section{3.- Generación de Código}

El proceso de desarrollo de las aplicaciones se realizó utilizando tecnologías web basadas en la filosofía de software libre, debido a que se siguen los lineamientos emitidos por el Ministerio de Industrias Básicas, Estratégicas y Socialista. Dentro de esta fase se generó el código necesario, haciendo uso del lenguaje de programación PHP. En el caso de la base de datos se realizó siguiendo las políticas descritas en el Manual Titulado Estándares de Base de Datos, usando como manejador MYSQL. 


\section{CONCLUSIONES Y APORTE DE LA INVESTIGACIÓN}

Para el diseño del Sistema de Información Web para el flujo de información entre VTELCA y la comunidad en general; se empleó en la codificación, herramientas basadas en estándares de software libre, lo cual permitió demostrar las bondades en cuanto a la generación de sistemas de información robustos, ágiles y flexibles, reduciendo de igual manera los costos generados en la producción de dicha aplicación.

Cabe considerar, que con el diseño de este sistema de información como estrategia tecnológica, incrementa la forma de capturar y procesar toda la información que maneja fábrica VTELCA para estas solicitudes y tramites, permitiendo la creación de nuevos procedimientos, normativas y manuales para solventar necesidades a nivel técnico, operativo, administrativo y sobre todo el envío de información a los solicitantes tenga un mejor control y seguimiento, de esta manera poder satisfacer a tiempo las necesidades de los mismos.

Finalmente, los resultados obtenidos en el diagnóstico de necesidades; reflejan la pertinencia del producto desarrollado en respuesta a los requerimientos especificados, permitiendo con ello evidenciar la eficiencia del sistema desarrollado.

\section{REFERENCIAS CONSULTADAS}

1. Arias, F. (2012), Proyecto de Investigación: Introducción a la Metodología Científica. 6ta Edición, Editorial Episteme. Caracas: Venezuela.

2. Gerencia de Operaciones (2012). Manual de Creación de Aplicaciones Informáticas. Punto Fijo: Venezolana de Telecomunicaciones C.A.

3. Hurtado, J. (2006). El proyecto de Investigación (4ta. ed.). Bogotá: Ediciones Quirón Sypal.

4. Kendall, K y Kendall, E. (2005). Análisis y Diseño de Sistemas (6a ed.). México. Editorial Pearson.

5. Lattá Arias, C. (2019). Uso de las TIC para proyectos productivos en las instituciones educativas del Municipio Zona Bananera. Magdalena. Colombia. Revista Arbitrada Interdisciplinaria Koinonía, 4(7), 233-246. doi:http://dx.doi.org/10.35381/r.k.v4i7.202 
6. Laudon y Laudon (2012) Sistema de Información Gerencial $12^{\circ}$ Edición.

7. Ley de infogobierno Gaceta Oficial de la República Bolivariana de Venezuela $\mathrm{N}^{\circ} 40.274$ Octubre de 2013.

8. Ley Orgánica de Ciencias, Tecnologías e Innovación Gaceta Oficial de la República Bolivariana de Venezuela №39.575 Diciembre 2010.

9. Ley Orgánica de Telecomunicaciones. Gaceta Oficial №36.970 de fecha 12 de Junio de 2000.

10. Montilva, J. (1999) Desarrollo de sistemas de información (2 $2^{\underline{a}}$ ed.). Mérida, Venezuela: Universidad de los Andes.

11. Universidad Pedagógica Experimental Libertador, Vicerrectorado de Investigación y Postgrado, (2003). Manual de trabajos de grado de especialización y maestría y tesis doctórales. ( $3^{\circ}$ ed.). Caracas: Fondo Editorial de Experimental Libertador.

\section{REFERENCES CONSULTED}

1. Arias, F. (2012), Research Project: Introduction to Scientific Methodology. 6th Edition, Editorial Episteme. Caracas Venezuela.

2. Hurtado, J. (2006). The Research project (4th ed.). Bogotá: Quirón Sypal editions.

3. Kendall, K and Kendall, E. (2005). Systems Analysis and Design (6th ed.). Mexico. Pearson editorial.

4. Lattá Arias, C. (2019). Use of ICT for productive projects in the educational institutions of the Municipality of Banana Zone. Cupcake Colombia. Interdisciplinary Arbitrated Review Koinonía, 4 (7), 233-246. doi: http: //dx.doi.org/10.35381/r.k.v4i7.202

5. Laudon and Laudon (2012) 12th Edition Management Information System.

6. Law of Official Government Gazette of the Bolivarian Republic of Venezuela No. 40,274 October 2013.

7. Libertador Experimental Pedagogical University, Vice Rectorate for Research and Postgraduate, (2003). Manual of works of degree of specialization and masters and doctoral thesis. (3rd ed.). Caracas: Experimental Libertador Publishing Fund. 
8. Montilva, J. (1999) Development of information systems (2nd ed.). Mérida, Venezuela: University of the Andes.

9. Operations Management (2012). Computer Applications Creation Manual. Fixed Point: Venezolana de Telecomunicaciones C.A.

10. Organic Law of Sciences, Technologies and Innovation Official Gazette of the Bolivarian Republic of Venezuela No. 39,575 December 2010.

11. Organic Law of Telecommunications. Official Gazette No. 36,970 dated June $12,2000$. 